\title{
Phase Separation and Electrical Resistivity of Liquid Cu-Co Alloys
}

\author{
Fengxiang Guo ${ }^{1,2, a}$, Wei Wang ${ }^{2,3}$, Qiwei Wang ${ }^{1}$, Jian Yuan ${ }^{1}$, Lei Li ${ }^{1}$, Huilue Jiang ${ }^{1}$, Zhonghai Zhou ${ }^{1}$ and Xuelei Tian ${ }^{2}$ \\ ${ }^{1}$ Shandong Provincial Key Laboratory of Ocean Environment Monitoring Technology, Institute of Oceanographic Instrumentation of \\ Shandong Academy of Sciences, Qingdao 266001, China \\ ${ }^{2}$ Key Laboratory for Liquid-Solid Structural Evolution and Processing of Materials (Ministry of Education), School of Material Science and \\ Engineering, Shandong University, 17923\#, Jingshi Road, Jinan 250061, China \\ 3 Institute of Hybrid Materials, Growing Base for State Laboratory, Qingdao University, 308\#, Ningxia Road, Qingdao 266071, China \\ ${ }^{a}$ Corresponding author
}

\begin{abstract}
Undercooling of $\mathrm{Cu}$-Co alloys is achieved with the help of glass flux technology. Electrical resistivity of $\mathrm{Cu}_{20} \mathrm{Co}_{80}$ and $\mathrm{Cu}_{60} \mathrm{Co}_{40}$ has been detected during the cooling process. In undercooled liquid, an inflection in resistivity is well accompanied by a turning point in temperature-time curve, which is believed a signature of phase separation in Cu-Co alloys. As the phase separation is in process, resistivity behaviours following Percolation model shortly. In as-solidified samples, SEM results indicate a separated microstructure.
\end{abstract}

\section{INTRODUCTION}

Melts structure has been attractive in both scientific and technological view of point. Local structure and atomic clusters take a predominant role in melts properties, and therefore of importance in as-solidified ingots. In facts, during the cooling process, most of the alloys would experience a stage of undercooling, due to the high nucleation barrier and difficulty. Then, more and more researches are focus on the structure and its transition in undercooled liquid state.

Early in 1995, the structure of liquid and undercooled Ge was studied by x-ray absorption experiments [1], however no evidence of structure transition was revealed. With the development of levitation technology, direct structure investigations are possible in bulk deeply undercooled liquid state. With the help of X-ray diffraction [2], the splitting in first peak and increasing covalent bonds were found in undercooled liquid $\mathrm{Si}$. Since the existence of icosahedral ordering was verified in liquid and undercooled $\mathrm{Cu}$ [3], more and more structure information is discovered, such as bond-angle distribution and fraction of nearly icosahedral configurations. Recently, the medium-range order arising from a strong tendency for $\mathrm{Pd}-\mathrm{Pd}$ avoidance is revealed in supercooled liquid $\mathrm{Zr}_{75.5} \mathrm{Pd}_{24.5}$ [4].

Although there is a lot of controversy, liquid-liquid phase transition in undercooled liquid has drawn more and more interest. A growing body of evidence has been reported in support of liquid-liquid phase transitions, from both experiments and and computer simulation. Combining small-angle and wide-angle x-ray scattering (WAXS), a first-order liquid/liquid phase transition in $\mathrm{Y}_{2} \mathrm{O}_{3}-\mathrm{A}_{2} \mathrm{O}_{3}$ melts was presented [5]. And a liquid-liquid phase transition was reported through simulation of supercooled liquid silicon [6], which is previously predicted by Aptekar.

Except for direct experimental evidence and simulation, structure sensitive physical properties are important path in the studies of undercooled liquid, such as electrical resistivity, magnetic susceptibility. In undercooled liquid Co-Pd alloys [7], the magnetic transition could not be obtained by direct structure investigation of EXAFS, due to the extreme undercooling.
However, the magnetic transition around Curie temperature was verified by magnetic measurement [8], which is believed to be a second-class phase change. Even the magnetic long range order could be revealed by the interaction [9] of Co-Pd melts with Co-Sm permanent magnet. Resistivity is a most important indicator of liquid-liquid structure transition in metallic melts. What's more, electrical resistivity [10] is also verified to be a sensitive indicator of the magnetic transition around Curie temperature. In this report, resistivity is used to characterize the liquid-liquid phase separation in undercooled $\mathrm{Cu}-\mathrm{Co}$ alloys.

\section{Experiments}

The resistivity measurements were performed from superheated liquid to the rapid solidification process in self-constructed equipment. Theoretically, the differential signal over the test coil and reference coil is zero if there is no sample in the test coil. The coil system with the sample would generate a voltage related to the resistivity of samples, which could be obtained as

$$
\left.\Delta U=K \mid \frac{\left|\mu_{\mathrm{eff}}\right|-\frac{\mu_{r 0}}{\mu_{r}}}{\left|\mu_{\mathrm{eff}}\right|-\frac{\mu_{r 0}}{\mu_{r}}+\frac{2 l S}{l_{s} S_{s}} \times \frac{\mu_{r 0}}{\mu_{r}}}\right) \mid
$$

where as proposed by Foster, effective permeability $\mu_{\text {eff }}$ could be obtained by

$$
\mu_{\mathrm{eff}}=\frac{2}{\sqrt{-\mathrm{i} \omega \mu_{0} r_{s}^{2} / \rho}} \frac{J_{1}\left(\sqrt{-\mathrm{i} \omega \mu_{0} r_{s}^{2} / \rho}\right)}{J_{0}\left(\sqrt{-\mathrm{i} \omega \mu_{0} r_{s}^{2} / \rho}\right)}
$$

$H$ and $\omega$ are intensity and angular frequency of periodic magnetic field, respectively. $\mu_{\mathrm{r} 0}, \mu_{\mathrm{r}}$ are the relative permeability of air and the sample, $K$ is a parameter dependent on the circuit and working frequency. The other symbols are the size of samples. Further details of the non-contact measurement device could be found in Ref. [11]. 
$\mathrm{Cu}(5 \mathrm{~N}), \mathrm{Co}(4 \mathrm{~N})$ were used in the non-contact measurement of electrical resistivity. The metals were pre-melted in induction furnace under $5 \mathrm{~N}$ Ar gas. The temperature calibration is made during a simultaneous detection by pyrometer (Raytek 3i) and S-type thermocouple, during the cooling process. The accuracy of temperature is about $\pm 5 \mathrm{~K}$. The metals were undercooled with the help of $\mathrm{B}_{2} \mathrm{O}_{3}$ flux. The evaluated absolute error in resistivity was no more than $3 \%$, because of the uncertainty in signal fluctuation and temperature error. The samples were also analysed by the TEM (200kV, JEM-2100).

\section{Results}

\subsection{Resistivity and temperature curves}

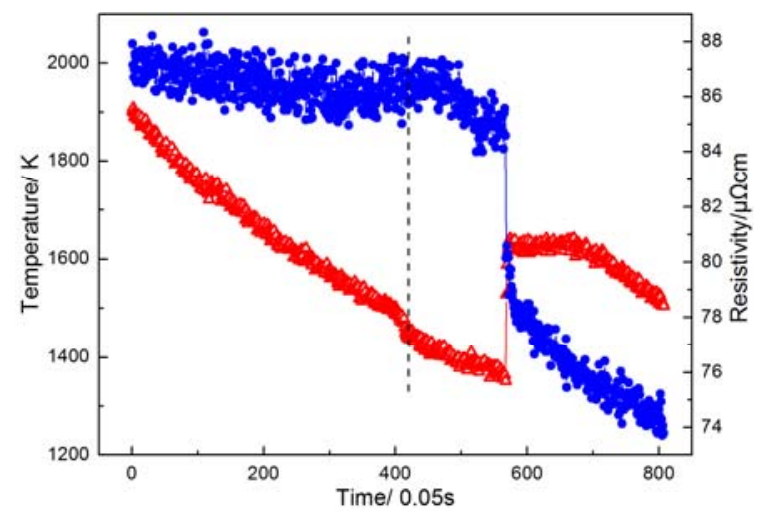

Figure 1. Resistivity (solid spheres) and temperature (open triangles) behaviour with time in $\mathrm{Cu}_{20} \mathrm{Co}_{80}$ alloys.

For $\mathrm{Cu}_{20} \mathrm{Co}_{80}$ alloys, the electrical resistivity and temperature during cooling process are shown in Figure 1. Obviously, resistivity and temperature decrease continuously till the time as indexed by the dashed line. Then there is an obvious inflection in the resistivity-time dependence. Simultaneously, a rapid drop emerges in the temperature-time curves. Then, resistivity decreases more rapidly as the time is in process till the recalescence time. During the recalescence process, an abrupt drop in resistivity is accompanied. The large recalescence speed indicates the rapid branch crystal growth under the large undercooling. After the recalescence, resistivity exhibits an exponential decrease as time processes, especially during the plateau after recalescence.

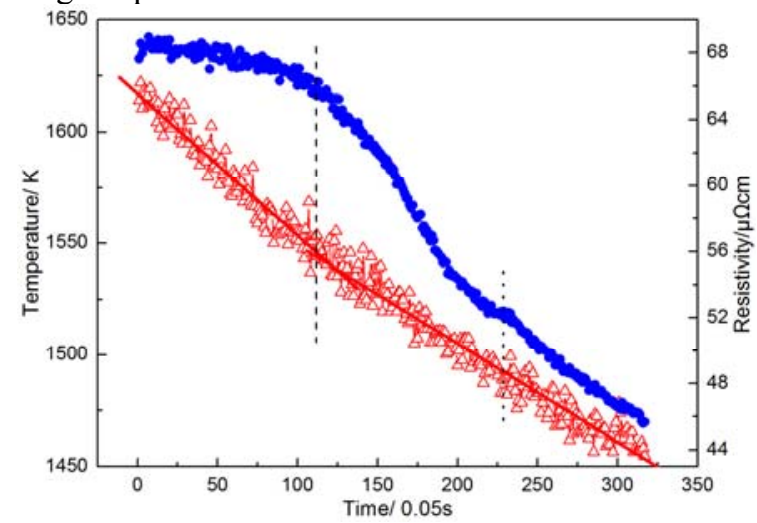

Figure 2. Resistivity (solid spheres) and temperature (open triangles) behaviour with time in $\mathrm{Cu}_{60} \mathrm{Co}_{40}$ alloys.
For $\mathrm{Cu}_{60} \mathrm{Co}_{40}$ alloys, the electrical resistivity and temperature during cooling process are deposited in Figure 2. Obviously, resistivity and temperature decrease almost linearly till the time as indexed by the dashed line. Then, there is an obvious upper inflection in the temperature-time curve. Simultaneously, a downward turning in resistivity emerges in the resistivity-time curves. It is worthy to be noticed that, in the time range that indexed by the dashed line and the dotted line, the temperature curves of resistivity seem to follow the percolation model. Then resistivity and temperature decrease continuously. Due to the relatively low undercooling, the recalescence is very weak and therefore is not exhibited here.

\subsection{Microstructure}

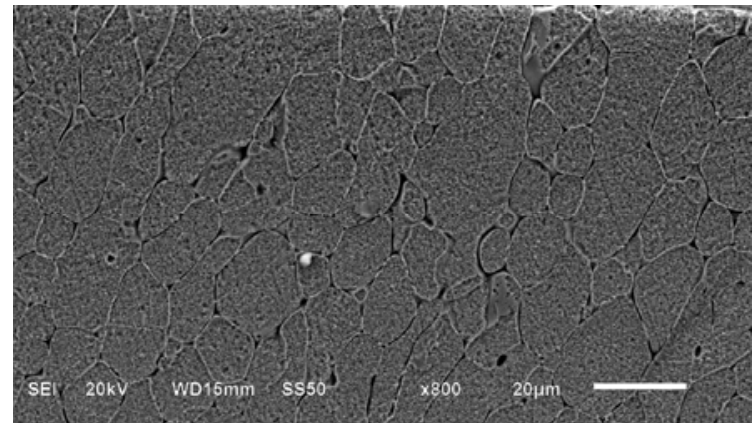

Figure 3. As-solidified microstructure of normal $\mathrm{Cu}_{20} \mathrm{Co}_{80}$

The normally as-solidified microstructure image of undercooled $\mathrm{Cu}_{60} \mathrm{Co}_{40}$ alloys is shown in Figure 3. For normal samples, obvious branch growth pattern is obtained with a grain radius of $15 \mu \mathrm{m}$. Most of the grains are non-regular with smooth grain boundaries.

While in the segregated alloys, there is a lot of $\mathrm{Cu}$ rich (light) phase surrounded by the Co-rich (dark) matrix phase, as shown in Figure 4. Most of the grain radius of the segregated $\mathrm{Cu}$-rich phase is less than $10 \mu \mathrm{m}$. And there are one or several Co-rich cores in the center of $\mathrm{Cu}$ rich phase. In this image, some $\mathrm{Cu}$-rich phases aggregate to form a large semi-sphere structure.

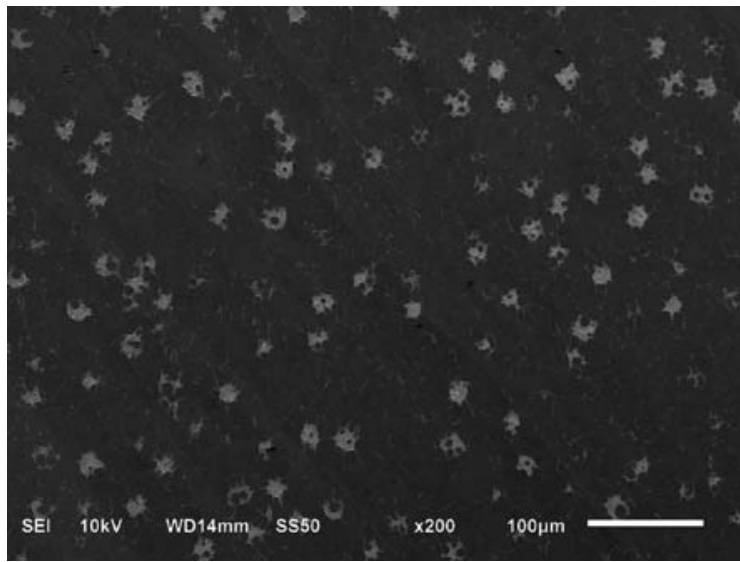

Figure 4. Microstructure of segregated $\mathrm{Cu}_{20} \mathrm{Co}_{80}$.

As shown in Figure 5, the microstructure images of segregated $\mathrm{Cu}_{60} \mathrm{Co}_{40}$ alloys are exhibited. There is a large Co-rich sphere in the center of the sample, which is the 
segregated phase in Cu-rich alloys. The boundary between $\mathrm{Co}$-rich phase and $\mathrm{Cu}$-rich matrix is quite clear, as deposited in Figure 5 (a). In the vicinity of the phase boundary, there are many second-class segregated spheres moving to the boundary. As shown in Figure 5 (b), the boundary of the second-class Co-rich phase is not smooth, due to the coagulation of the other segregated Co-rich sphere inside the Cu-rich matrix. Similarly, in the segregated Co-rich phase, many $\mathrm{Cu}$-rich spheres with fuzzy boundary are observed.

\section{Discussions}

Resistivity and temperature behaviour with time exhibit a good agreement both in $\mathrm{Cu}_{20} \mathrm{Co}_{80}$ and $\mathrm{Cu}_{60} \mathrm{Co}_{40}$. Combined with the as-solidified microstructure, the inflection in resistivity is verified to be the signature of liquid-liquid phase segregation in undercooled liquid $\mathrm{Cu}-$ $\mathrm{Co}$ alloys [12]. During the segregation process of $\mathrm{Cu}-\mathrm{Co}$ alloys, gravity and surface tension are the phaseseparation driving force. Phase separation could only be described by the simultaneous presence of nucleation, growth, and coagulation rather than traditional theories of diffusional growth. Then, as segregation processes, $\mathrm{Cu}-$ rich phase with low resistivity coagulates and provides preferential path for electron mobility. Then, resistivity decreases during the segregation and coagulation process till the recalescence. During recalescence process of $\mathrm{Cu}_{20} \mathrm{Co}_{80}$, a large percentage of melts solidify for the high recalescence velocity, which results in the rapid drop in resistivity. After recalescence, as the solidification processes, the resistivity of the sample decreases rapidly.

In undercooled $\mathrm{Cu}_{60} \mathrm{Co}_{40}$ melts, $\mathrm{Cu}$-rich phase and Co-rich are the matrix and segregation phase. Then, as segregation processes, $\mathrm{Cu}$-rich with low surface tension and larger percentage would move toward to the surface of the samples, to decrease the total energy of the samples to the minimum. Indeed at the early stage, the nucleation of segregation and coagulation is quite slow. Then as segregation coagulates, the coagulation speed and resistivity decrease become more and more quickly. However, the temperature and the viscosity increase in the meantime, which results in the more difficult coagulation and slower decrease in resistivity. Then, the resistivity decreases with decreasing temperature till the segregation is slow enough. There, during the segregation process, the resistivity of $\mathrm{Cu}_{60} \mathrm{Co}_{40}$ follows a Percolation model.
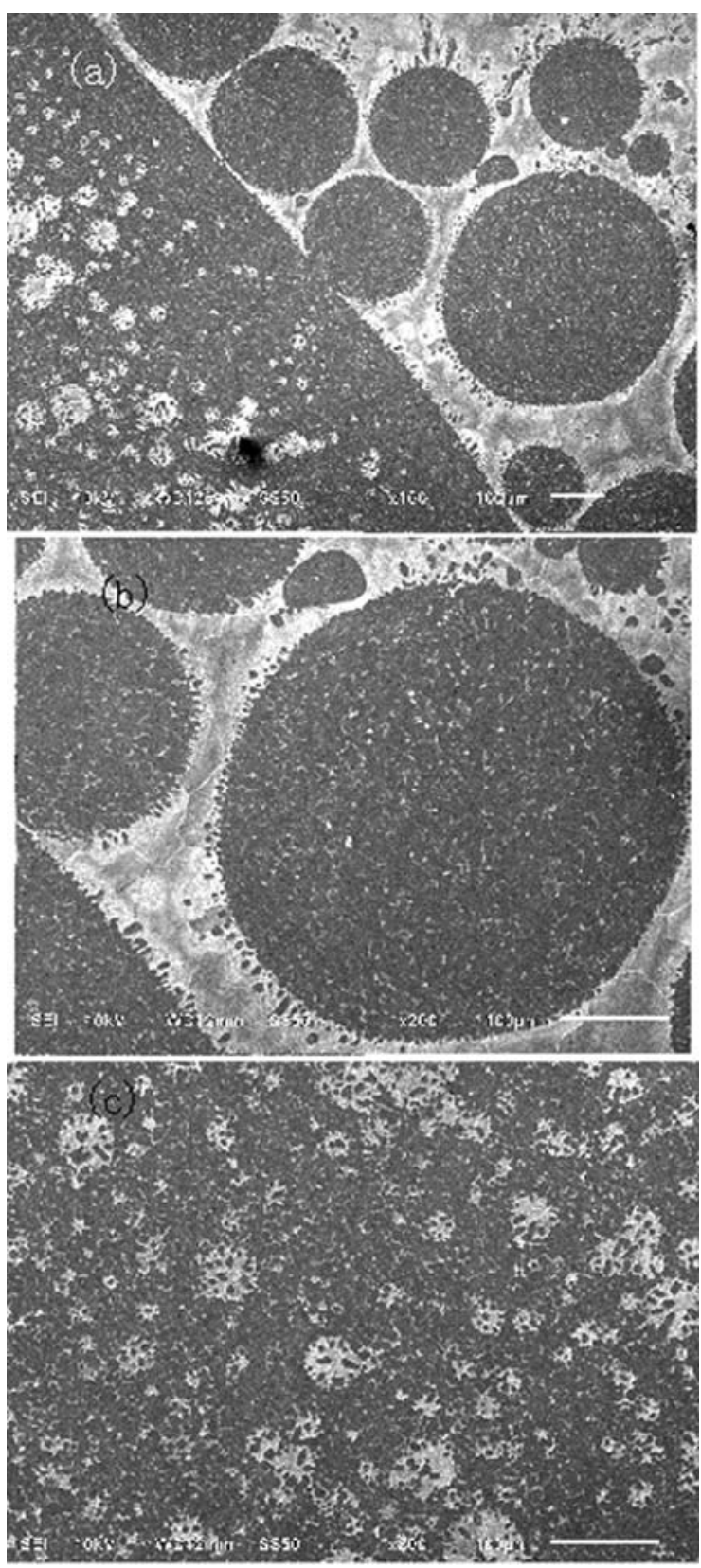

Figure 5. Microstructure of segregated $\mathrm{Cu}_{60} \mathrm{Co}_{40}$ alloys. a, segregation phase boundary, b, second class Co-rich phase, c, second class $\mathrm{Cu}$-rich phase.

In good agreement with the resistivity behaviour, the microstructure indicates the existence of phase separation. As it is shown, the $\mathrm{Cu}$-rich and Co-rich phases are the segregation phase in $\mathrm{Cu}_{20} \mathrm{Co}_{80}$ and $\mathrm{Cu}_{60} \mathrm{Co}_{40}$ alloys [13]. There exists the second and higher class separation in the segregation and matrix of $\mathrm{Cu}_{60} \mathrm{Co}_{40}$, for the relatively low undercooling. This verifies the role of surface tension in the segregation. However, the undercooling is too large and the Co-rich matrix phase nucleates at the early stage of the phase segregation in $\mathrm{Cu}_{20} \mathrm{Co}_{80}$. As shown in Figure 1 , the rapid segregation lasts shortly in undercooled liquid $\mathrm{Cu}_{20} \mathrm{Co}_{80}$ alloys. Therefore no large $\mathrm{Cu}$-rich phase is obtained, even if the coagulation process is observed. 


\section{Conclusions}

Resistivity of liquid $\mathrm{Cu}-\mathrm{Co}$ alloys has been verified to be effective as an indicator of metastable phase segregation in undercooled liquid state, which is indicated by the assolidified microstructure. The $\mathrm{Cu}$-rich and Co-rich phases are the segregation phase in $\mathrm{Cu}_{20} \mathrm{Co}_{80}$ and $\mathrm{Cu}_{60} \mathrm{Co}_{40}$, respectively.

\section{References}

[1] A. Filipponi, Phys. Rev. B 51 12322(1995)

[2] S. Ansell, S. Krishnan, J.J. Felten, D.L. Pricey, J. Phys.: Condens. Matter. 10 L73-L7(1998)

[3] A. Di Cicco, A. Trapananti, S. Faggioni, A. Filipponi, Phys. Rev. Lett. 91, 135505(2003)

[4] N.A. Mauro, W. Fu, J.C. Bendert, Y.Q. Cheng, E. Ma, K.F. Kelton, J. Chem. Phys. 137, 044501(2012)

[5] G.N. Greaves, M.C. Wilding, S. Fearn, D. Langstaff, F. Kargl, S. Cox, Q. Vu Van, O. Majérus, C.J. Benmore, R. Weber, C.M. Martin, L. Hennet, Science 322 566(2008)

[6] S. Sastry, C.A. Angell, Nature Materials 2, 739(2003)

[7] G. Jacobs, I. Egry, D. Holland-Moritz, D. Platzek, J Non-Cryst. Solids 232-234 396 (1998)

[8] S. Reutzel, D.M. Herlach, Mater. Sci. Eng. A 375377 552(2004)

[9] D. Platzek, C. Notthoff, D.M. Herlach, G. Jacobs, D. Herlach, K. Maier, Appl. Phys. Lett. 65, 1723(1994)

[10] G. Lohofer, S. Schneider, I. Egry, Int J Thermophys, 22, 593(2001)

[11] M.X. Zhang, X.L. Tian, F.X. Guo, Acta Phys Sin Ch Ed, 58 6080(2009)

[12] C.D. Cao, G. Gorler, D. Herlach, B. Wei, Mater. Sci. Eng. A 325 503(2002)

[13]F. Guo, T. Lu, J. Qin, H. Zheng, X. Tian, Physica B 407 4108(2012) 